J. Lake Sci. (湖泊科学) , 2019, 31(3): 609-620

DOI 10. 18307/2019. 0301

(c) 2019 by Journal of Lake Sciences

\title{
冰川融水对山地冰冻圈冰湖水文效应的影响”
}

\author{
王 欣 ${ }^{1,2}$, 丁永建 ${ }^{2 * *}$, 张 勇 ${ }^{1,2}$ \\ ( 1 : 湖南科技大学资源环境与安全工程学院, 湘潭 411201) \\ (2: 中国科学院西北生态环境资源研究院冰冻圈科学国家重点实验室,兰州 730000)
}

摘 要: 冰川融水通过热量、水、物质传输对山地冰冻圈冰湖水文效应产生影响, 引起广泛关注. 本文从山地冰冻圈冰湖 的水量、物理化学性质、生物等方面系统总结冰川融水对冰湖水文效应的影响. 冰川融水被冰湖滞留能在一定程度上延 缓区域冰川水资源的亏损, 但也直接导致了潜在危险性冰湖数量和危险程度增大. 冰川融水对冰湖物理性质的影响主要 表现在降低湖水温度、影响透明度/浊度、改变湖水密度、造成湖水热力分层现象等方面,对冰湖化学性质的影响主要表 现在增加湖水中的氮素、溶解有机物、持久性有机污染物、各类离子和重金属等,进而影响冰湖生物的分布、组成、结构和 功能. 深人系统地开展冰川融水及其变化对冰湖水文效应研究, 对冰川水文与水资源、山地冰冻圈生态环境研究具有重 要意义.

关键词: 山地冰冻圈;物理化学性质;冰湖;水文效应; 灾害

\section{The influence of glacier meltwater on the hydrological effect of glacial lakes in Mountain Cryosphere}

\author{
WANG Xin ${ }^{1,2}$, DING Yongjian ${ }^{2 * *}$ \& ZHANG Yong ${ }^{1,2}$ \\ (1: School of Resource Environment and Safety Engineering, Hunan University of Science and Technology, Xiangtan 411201, \\ P.R. China) \\ (2: State Key Laboratory of Cryospheric Science, Northwest Institute of Ecology and Environmental Resources, Chinese Acade- \\ my of Sciences, Lanzhou 730000 , P.R. China)
}

\begin{abstract}
Much attention has been attracted on the glacier meltwater due to its influence on the hydrological effect of glacial lakes through heat, water and material transmission in Mountain Cryosphere. We summarized the impacts of glacier meltwater on the hydrological effects with respects to the water quantity, physicochemical properties and biology of glacial lake in this paper. A certain amount of glacier water resources has been temporarily held resulted by the meltwater direct inflowing into glacial lakes, which retarded the loss water resources in the glaciated area to some extent on one hand, increased the number and hazardousness of the potential dangerous glacial lakes on the other hand. Glacier meltwater affects both physical and chemical properties of glacial lakes. Meltwater normally reduced lake temperature, disturbed lake transparency/turbidity, and facilitated thermal stratification of the glacial lake. Meltwater also increased the contents of nitrogen, dissolved organic matter, persistent organic pollutants, ion concentrations and heavy metals in the glacial lake water, influenced the distribution, composition, structure and function of glacial lake organisms and ecosystem. An in-depth and systematic research on the impacts of glacier meltwater and its changes on hydrological effect of glacial lakes, is of great significant to the glacier hydrology, water resources and ecological environment study of Mountain Cryosphere.
\end{abstract}

Keywords: Mountain Cryosphere; physical chemistry properties; glacial lake; hydrological effect; hazard

冰湖 (与冰川或冰川作用相关的湖泊 ${ }^{[1]}$ ) 在世界各地山地冰冻圈广泛发育, 受地形和补给源的影响, 一

* 国家自然科学基金项目 (41771075,41271091) 和国家重大科学研究计划重大科学目标导向项目 (2013CBA01800) 联合资助. 2018-08-31 收稿;2018-11-11 收修改稿. 王欣(1973 ), 男,博士, 教授; E-mail: xinwang_hn@ 163.com.

** 通信作者; E-mail: dyj@lzb.ac.cn. 
般规模不大 (如我国冰湖规模在 $0.0036 \sim 5.83 \mathrm{~km}^{2}$ 之间 ${ }^{[2]}$ ) 且多受现代冰川融水补给, 其分布的数量和规模 与冰川分布的数量和规模密切相关. 最近的冰湖遥感调查显示, 2010 年在亚洲中部以青藏高原为中心的高 海拔区域(简称高亚洲, 主要包括喜马拉雅山、念青唐古拉山、昆仑山、喀喇昆仑山、天山等山系)共发育 7368 个冰湖, 总面积为 $779 \mathrm{~km}^{2}$, 其中现代冰川融水直接补给的冰湖约占总数量的 $78 \%$ 和总面积的 $83 \%{ }^{[3-4]}$. 由于 山地冰冻圈特殊的地理环境及水文特征, 使得山地冰冻圈内环境变化对冰湖水文效应的影响以及冰湖水文 对环境变化的响应都具有特殊性.

在当前山地冰川普遍退缩、冰川物质负平衡加剧的背景下, 冰湖与冰川补给湖面积呈现出普遍扩张的 态势 ${ }^{[5]}$, 这不仅影响山地冰冻圈的水文循环过程, 而且还改变了其物理、化学和生物特性 ${ }^{[6]}$. 山地冰冻圈对 气候变化敏感,而位于其中的冰湖更是区域气候变化的极佳指示器 ${ }^{[7-8]}$, 进一步了解冰川融水对冰湖水文的 过去、现在和未来产生的影响具有重要意义. 山地冰冻圈的变化及其影响受到广泛的关注 ${ }^{[9-14]}$, 众多研究在 关注冰冻圈要素 (冰川、冻土、积雪) 变化水文效应的同时, 也注意到了冰川融水对冰湖的影响 ${ }^{[15-18]}$, 然而当 前仍缺乏冰川变化与冰湖水文效应关系研究的系统总结与展望. 本文基于近年来的研究成果, 从冰湖水循 环、水文过程、水文情势及水环境等角度, 系统总结了冰川融水对冰湖水量、物理化学性质、生物等产生的水 文效应及其影响, 提出了冰川融水对湖泊水文效应影响的概念模型 (图 1), 展望了开展冰川融水对冰湖水文 效应研究问题及前景.

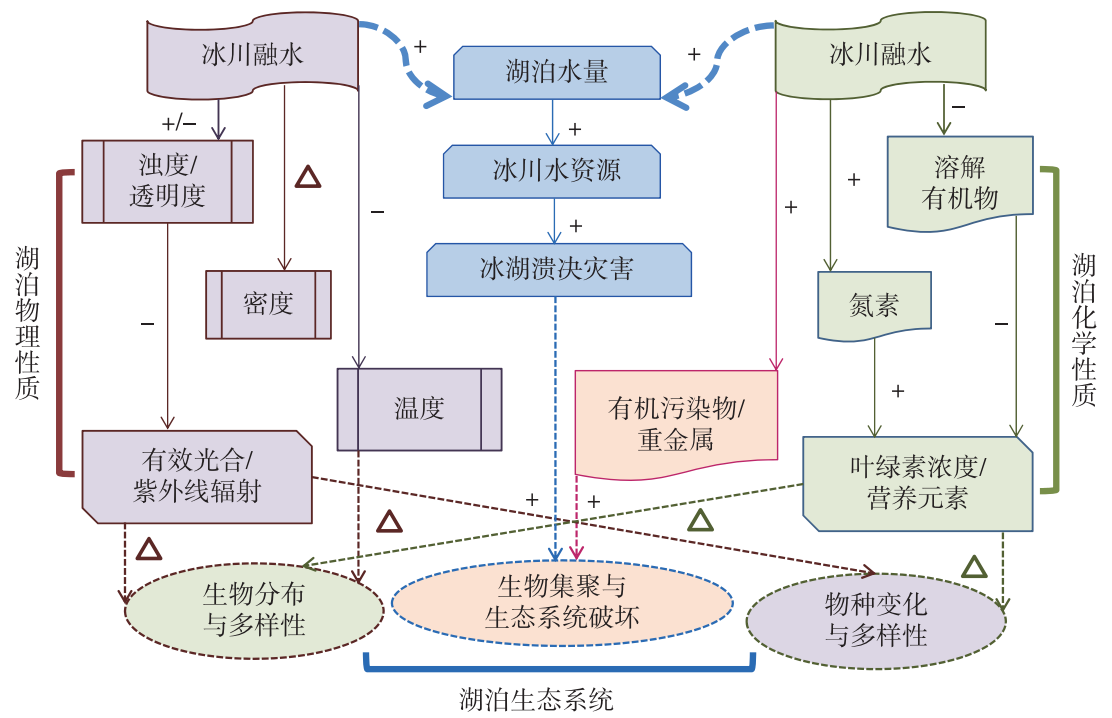

图 1 冰川融水对山地冰冻圈湖泊水文效应影响的概念模型( “+”表示增加, “-”表示减少, “ $\triangle ”$ 表示变化)

Fig. 1 Conceptual model of the glacier meltwater impacts on the hydrological effect of the glacial lake in Mountain Cryosphere ( “+” indicates an increase, “-” indicates a decrease, and “ $\triangle$ ” indicates changes)

\section{1 冰川融水与冰湖水量}

\section{1 冰湖水量平衡}

山地冰冻圈的冰湖在其形成发育期多为封闭的内陆湖, 人湖水量主要由降水和冰川消融补给, 支出水 量主要由蒸发构成,在单位时间内,其水量平衡方程可表示为:

$$
\Delta H=P+R_{\mathrm{S}}+R_{\mathrm{g}}-E \pm \varepsilon
$$

式中, $\Delta H$ 为冰湖水变化量, $P$ 为湖面的年降水量, $R_{\mathrm{S}}$ 为由降水产生的地表径流折算成的径流深度, $R_{\mathrm{g}}$ 为冰川 融水的补给量, $E$ 为湖面的蒸发量, $\pm \varepsilon$ 为冻土融水、地下水、渗流作用导致的变化量. 融水对湖水量的影响可 分为短时间 (日)、中等 (季节) 和长时间尺度 (年际、年代际甚至更长), 一般来说, 在地理环境变化不大的情 景下, 冰湖水量的收人和支出处于动态平衡状态, 即蓄水变化量为零. 
一般冰湖的水量/面积变化对冰川变化尤其敏感,过去几十年来,高亚洲、阿尔泰山、安第斯山、阿尔卑 斯山、巴塔哥尼亚高原等地总体呈现冰川普遍退缩、冰湖面积扩张的趋势. 如高亚洲众多冰川普遍退缩、冰 川物质负平衡加剧, 西昆仑和喀喇昆仑山地区冰川 物质则略微有正平衡趋势或者保持相对稳定 ${ }^{[19-22]}$. 与区域冰川差异性变化对应, 冰湖水量变化也呈现 显著的区域差异. 现有的研究结果显示, 自 1970s1980s 以来,念青唐古拉西部地区冰湖平均扩张速 度最大, 达 $4.67 \% \mathrm{a}^{-1[23]}$, 喜马拉雅山中东段冰湖面 积平均增速次之, 为 $1 \% \sim 3 \% \mathrm{a}^{-1}[24-26]$, 天山地区为 $0.8 \% \mathrm{a}^{-1[4]}$, 帕米尔为 $0.45 \% \mathrm{a}^{-1[7]}$, 横断山区为 $0.12 \% \mathrm{a}^{-1[27]}$, 而在喀喇昆仑和兴都库什山脉部分区 域冰湖面积甚至以 $1.5 \% \sim 2.5 \% \mathrm{a}^{-1}$ 的速率减少 ${ }^{[24]}$. 海拔上, 分析珠峰地区不同高度带内冰川与冰湖面 积变化率显示, 冰川退缩的峰值高度 $5700 \sim 5900 \mathrm{~m}$ $(-33.5 \%)$ 和 $5200 \sim 5400 \mathrm{~m}(-26.5 \%)$ 与冰湖面积增 长的两个峰值高度 $5400 \sim 5600 \mathrm{~m}(+47.6 \%)$ 和 5000 $5300 \mathrm{~m}(+49.8 \%)$ 对应 (图 2), 说明冰川融水对其下 游 100 300 $\mathrm{m}$ 的冰湖面积扩张贡献明显 ${ }^{[28]}$.

可见,尽管由于冰川退缩、降水变化、湖盆形态

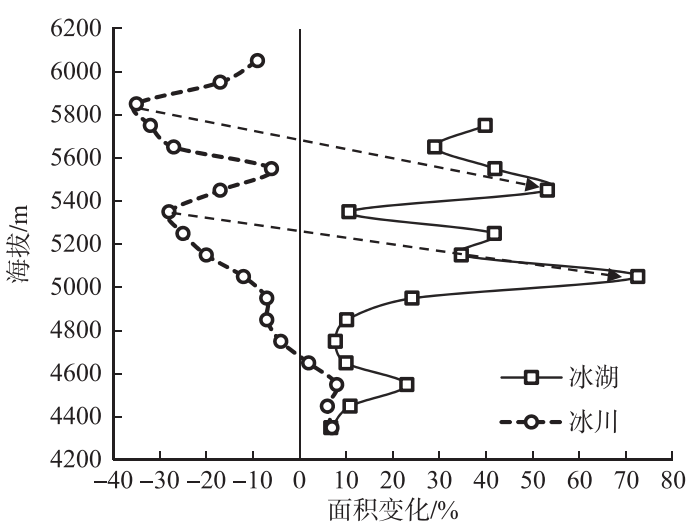

图 2 珠峰地区不同高度带冰川面积退缩与 冰湖面积扩张对比 ${ }^{[28]}$

Fig. 2 Glacier area retreat and glacial lake area expansion of different evelation bands in Everest region ${ }^{[28]}$

因素等的差异导致冰川融水与冰湖扩张之间耦合关系复杂 ${ }^{[29-31]}$, 但是山区冰川物质平衡强烈亏损对冰湖水 量变化具有重要影响,主要表现为冰川融水对于近期冰湖水量增加的补给作用 ${ }^{[3,16,23,32-33]}$.

\section{2 冰湖水资源及灾害}

冰川融水注人冰湖导致其面积增加的现象, 说明至少部分冰川水资源并没有通过地表随径流系统流出 冰冻圈, 而是被冰湖暂时滞留, 这在一定程度上调整了山区的水循环过程, 延缓了因气候变暖而导致区域冰 川水资源的亏损 ${ }^{[4,34]}$ (图 1), 影响冰湖的蓄水量和蓄水周期甚至海平面的变化 ${ }^{[35]}$. 因此, 研究者开始关注山 地冰冻圈冰湖储存了多少冰川水资源, 或者说, 冰湖能在多大程度上延缓气候变暖引起的冰川水资源亏损. 在天山, 2010 年冰川融水直接补给的冰湖水储量约为 $1.78 \mathrm{Gt}, 2000-2010$ 年, 每年大约有 $2 \%$ 的冰川融水储 存在冰湖中, 致使天山冰湖总水量增率约为 $0.01 \mathrm{Gt} / \mathrm{a}^{[4]}$. 这部分被冰湖滞留融水的比例不大, 但对内陆干旱 区的生态和社会经济建设极为珍贵.

对于山地冰冻圈中以冰川融水补给为主的冰湖, 由于冰川融水被冰湖滞留而在湖中聚集, 湖盆原有的 水循环平衡状态被破坏, 冰湖的潜能 (冰湖水量、湖水比重与堤坝高的乘积) ${ }^{[37]}$ 和潜在溃决洪水体积 (假设 湖泊在堤坝背水坡坡度 $>10^{\circ}$ 的部分全面被冲毁后释放的水量 $)^{[38]}$ 都将增加, 从而导致潜在危险性冰湖数量 和危险程度增大, 如在中国喜马拉雅山地区, 该类潜在危险性冰湖达 142 个 $^{[36]}$ 、天山为 60 个 $^{[4]}$ (图 3), 预测 显示从 2020s 至 22 世纪是冰湖溃决灾害高发期 ${ }^{[39]}$. 另一方面, 融水被冰湖滞留的同时, 也在一定程度上改 变了湖盆原有能量传输路径, 由于水的反照率小, 湖盆水量的增加也意味着对太阳辐射吸收的增强, 热量在 冰湖中积聚. 山地冰冻圈冰湖堤坝多为冻土, 内有埋藏冰, 在湖中积聚的热量将会加快堤坝冻土消融, 致使 坝体稳定性下降 ${ }^{[40]}$. 以冰川阻塞形成麦兹巴赫湖, 在冰川融水蓄积达到一定水深 (或者水压) 时, 形成周期 性突发洪水 ${ }^{[41-42]}$. 可见, 冰川融水在短时间内汇聚到冰湖中, 冰湖静水压力增大, 是漫顶冲刷、管涌、冰坝抬 升、埋藏冰消融等冰湖溃决机制的诱因.

\section{2 冰川融水与冰湖理化性质}

\section{1 冰湖物理性质}

冰川融水对冰湖物理性质的影响主要表现在对湖水温度/密度、湖水分层/运动、透明度/浊度等方面 (图 1). 因冰川融水 (接近 $0^{\circ} \mathrm{C}$ ) 的输人, 致使冰川融水补给湖的平均水温度普遍较无冰川补给的水温低 2 

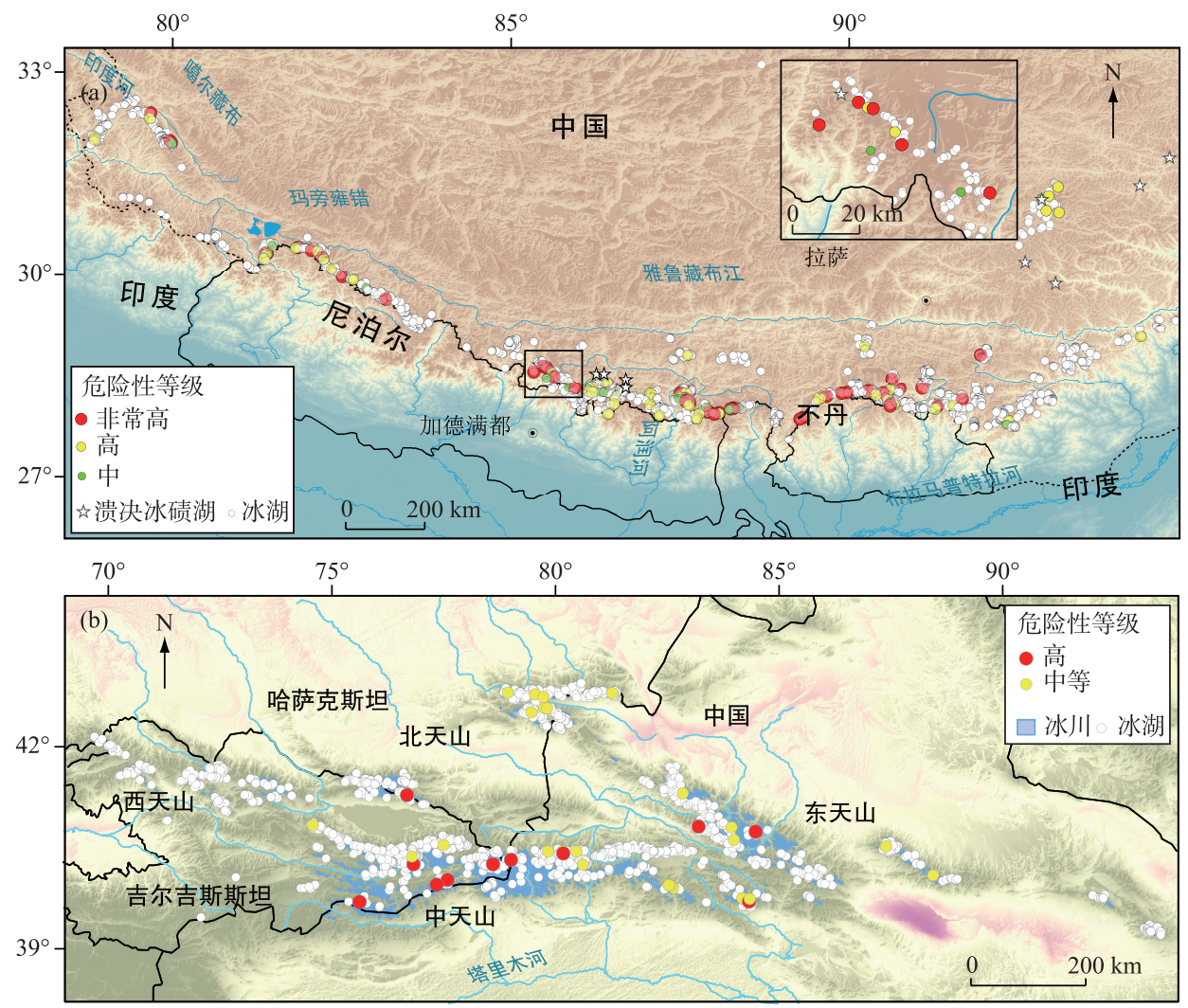

图 3 因冰川融水不断注人而形成的不同危险性等级的潜在危险性冰湖分布 ( $\mathrm{a}$ :中国喜马拉雅山 ${ }^{[36]} ; \mathrm{b}$ : 天山 ${ }^{[4]}$ )

Fig.3 Distribution of potential dangerous lakes of different outburst probability levels fed by glacier meltwater (a: in Himalaya, China ${ }^{[36]}$; b: in Tienshan mountain ${ }^{[4]}$ )

$3^{\circ} \mathrm{C}^{[43]}$, 两者温度差的大小与冰川融水补给比重、与冰川远近等密切相关; 如冰川融水叠加降水等影响, 湖水 温度呈现出独有季节和日动态变化, 表现出非连续、低温、多模式循环变化 ${ }^{[4]}$. 流域冰川覆盖度直接决定冰 川融水对冰湖的补给量和补给比例, 影响湖水温度, 进而影响冰湖生态系统 ${ }^{[45-46]}$. 研究发现, 流域内冰川覆 盖度与水温呈显著负相关, 冰川覆盖率每减少 $10 \%$ 水温可升高 $1.6^{\circ} \mathrm{C}^{[47]}$, 而冰川覆盖率每升高 $10 \%$, 夏季 (7-9 月) 湖水水温将降低 $0.6 \sim 2.0^{\circ} \mathrm{C}^{[48]}$. 此外, 冰川融水还影响着湖水温层的垂直分布和差异, 使得冰湖水 温变化更加复杂, 如美国的 Coghill 冰湖, 在水深 $1 \mathrm{~m}$ 处且据冰川补给源近的水域温度相对于同一深度距离 较远水域要低 $0.5 \sim 1.0^{\circ} \mathrm{C}$, 而在该湖更深的水层中却不存在这种温度差异 ${ }^{[43]}$; 巴塔哥尼亚冰原南部和智利中 部, 冰川融水汇人冰湖底层, 降低深层湖水温度使得湖水表层与深层温差达 $3 \sim 10^{\circ} \mathrm{C}^{[49-50]}$; 在奥地利阿尔卑 斯山,冰川补给湖表层水温日变幅是非冰川融水补给湖的 $1.3 \sim 1.5$ 倍 ${ }^{[44]}$.

冰湖的湖水密度主要受温度控制, 在 $4^{\circ} \mathrm{C}$ 左右时密度最大, 当水温高于或低于 $4^{\circ} \mathrm{C}$ 时, 密度变小. 夏季如 接近 $0^{\circ} \mathrm{C}$ 冰雪融水直接汇人冰湖, 冰湖表层水温会因与 $0^{\circ} \mathrm{C}$ 冰雪融水混合而改变, 从而导致上下层湖水密度 的差异, 形成湖水的分层与翻转现象 ${ }^{[51-52]}$. 如基于器测数据显示, 在天山托木尔峰的冰面湖, 夏季白天因冰 面气温上升消融, 接近 $0^{\circ} \mathrm{C}$ 的融水注人湖中, 与相对高温湖面水混合到 $4^{\circ} \mathrm{C}$ 左右, 因密度大而流向湖底, 产生 因动力下沉而形成湖水日垂直流, 14:00-16:00 左右在深层湖水形成低温谷 (图 4). 这种白天因融水注人 而在湖底形成低温谷的机制, 在一定呈上抑制了因太阳辐射导致的湖水温度上升 ${ }^{[29]}$. 但是, 这种湖水由热 力分层到动力翻转现象与融水汇人量的阈值条件尚需深人研究. 

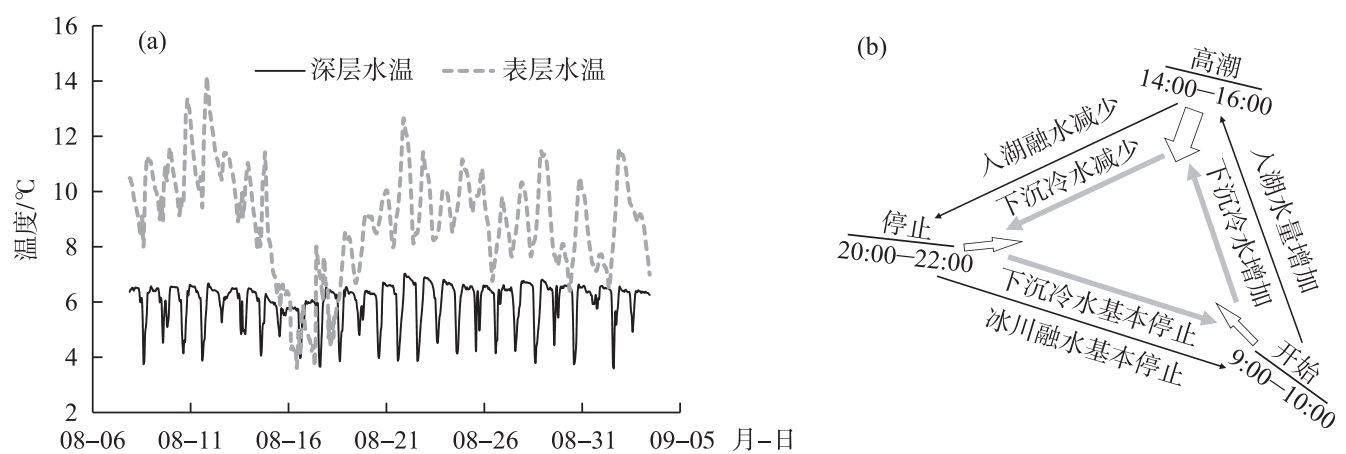

图 4 天山托木尔峰的冰面湖表层水温与深层水温变化差异 (a) 以及深层日低温谷形成示意图 (b) ${ }^{[29]}$

Fig.4 Difference between surface water temperature and deep water temperature of supraglacial lake in

Mount Tomer, Tienshan mountain (a), and schematic diagram showing the forming process of daily low temperature in the deep layer of supraglacial lake $(b)^{[29]}$

冰川融水通过冰面流携带表碛物质和冰下水流侵蚀底部基岩/排水通道携带的物悬浮质进人湖中, 影 响湖水的透明度/浊度, 冰川融水中的悬浮质粒径多在 $0.7 \sim 4.0 \mu \mathrm{m}$ 之间 ${ }^{[53]}$. 湖水的透明度/浊度随着冰川融 水的变化而存在时间和空间上的差异,随融水进人湖水中的悬浮质可使湖水光合有效辐射 (PAR) 和紫外线 (UVR) 的衰减量增大 $20 \% \sim 25 \%{ }^{[54-55]}$, 并周期性地驱动湖水表层温度增加和限制能量向深层传输 ${ }^{[44]}$. 一般 冰川融水直接补给、新生成的冰湖较为浑浊, 水体颜色呈灰色 (图 5a), 对光表现出强烈的散射和微弱的吸收 特性 ${ }^{[56]}$; 随着冰川退缩和冰湖的演化, 悬浮物质沉积, 浊度减小, 藻类生物的光合作用效率提高, 湖水呈现绿 色 (图 5b); 当冰川消失, 冰川融水不再直接补给冰湖时, 湖水往往呈现深蓝色 (图 5c), 对光表现出强烈的吸 收和微弱的散射特性 ${ }^{[56]}$. 湖水透明度/浊度是多因素综合的结果, 湖与冰川的距离、冰川径流的季节性差 异、湖水的深度、流动性、热力/盐度分层、风场的改变 (底泥再悬浮)、融水对湖底沉积物的物理扰动等均可 能影响湖水透明度/浊度的变化 ${ }^{[6]}$, 但冰湖与冰川的距离对湖水透明度/浊度的影响最直接 ${ }^{[6,57-59]}$.
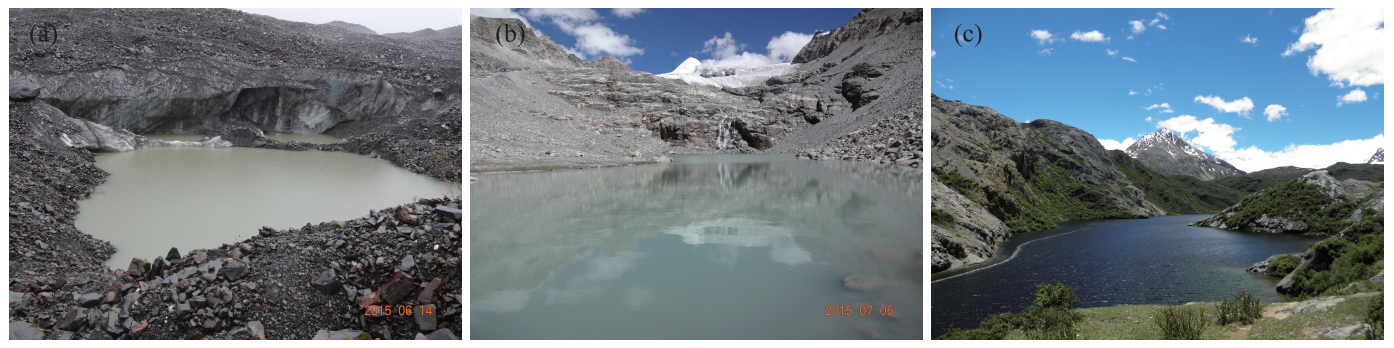

图 5 不同类型湖水透明度/浊度差异:新形成的冰面湖 (a)、

冰川融水直接补给但不直接临近冰川湖 ( b ) 和非冰川融水直接补给湖 (c)

Fig.5 Difference of water transparency/turbidity between different type lakes: new-formed supraglacial lake (a), glacier-fed lake without contact with glacier (b) and non-glacier-fed lake (c)

\section{2 冰湖化学性质}

冰川融水对冰湖化学性质的影响主要表现在对湖水中的氮素(氮氧化物及硝酸盐、亚硝酸盐、铵盐等化 合物)、溶解有机物 (DOM) 、持久性有机污染物 (POPs)、各类离子浓度和重金属含量等的影响 (图 1), 影响 强度与湖盆区的大气环流、地形地貌特征及冰湖一冰川间距离等有关.

1) 冰湖中氮素和 DOM. 对以冰川融水补给为主的冰湖, 湖水中的氮素主要来源于保存在冰内的大气沉 降氮、冰内氨化作用和硝化作用形成的氮等 ${ }^{[60]}$. 在冰川融水补给系统中, DOM 相对来源久远 (600 8000 
年) 且不稳定 ${ }^{[61-62]}$. 冰川径流变化影响 DOM 的输出及其含量, 在阿拉斯加山地冰川区, 冰川覆盖率低的流域 日溶解有机碳含量高 ${ }^{[46]}$. 由于冰川作用区较低的植被覆盖度减少陆地生态环境对氮素的摄人, 冰湖能从冰 川融水中获得更多的氮素, 使得湖水中氮的浓度相对较高, 尤其是 $\mathrm{NO}_{3}^{-}$浓度要高出一般积雪补给湖泊 $\mathrm{NO}_{3}^{-}$ 浓度的 $1 \sim 2$ 数量级 ${ }^{[59,63]}$. 高海拔区积雪的覆盖使得植物减少了氮素的利用, 加强了土壤中微生物过程, 当 积雪厚度较深时, 湖水中氮素的浓度更高 ${ }^{[64]}$. 所以, 山地冰冻圈冰湖中的氮素浓度高主要是因为补给湖泊 的冰川融水与土壤植被接触少, 相对于湖泊的其他补给源来说, 其同化和反硝化作用弱、损耗低 ${ }^{[60]}$.

2) 冰湖中 POPs. 冰冻圈是全球 POPs 重要的集聚地之一, 研究表明雪能有效地从空气中凝集以气相存 在和吸附于颗粒表面上的 POPs, 降雪的过程就是污染物的湿沉降过程, 冰川区的低温、低有机物质含量、渠 道化径流等特征有利于 POPs 的积累 ${ }^{[65-66]}$. 冰川通过融水把这些污染物带人冰湖中, 并在冰湖生物和湖底沉 积物中积累 ${ }^{[67-68]}$, 而气候变暖和冰川退缩将直接促进这一过程 ${ }^{[69]}$, 使得冰川成为 POPs 的二次污染源 ${ }^{[07-71]}$. 如在北美洲, 冰川融水补给的冰湖中 POPs 浓度至少是非冰川融水补给的湖泊中的 6 倍左右, 其中冰湖中 $50 \% \sim 97 \%$ 的 POPs 是由冰川融水贡献的 ${ }^{[65]}$; 在意大利阿尔卑斯山, 冰川融水补给湖湖底沉积物中有机氯类 杀虫剂 DDT 的峰值出现在冰川开始大量融化的 $1990 \mathrm{~s}^{[67,72]}$, 而非冰川融水补给湖中 DDT 的峰值则出现在 1970 s, 即 DDT 曾大量使用的时代 ${ }^{[73]}$.

3) 冰湖中离子浓度和重金属含量. 冰川的快速消融不仅增强了无机和有机物质的迁移, 还增强了冰川 融水与基岩和土壤的相互作用, 导致冰湖中各类离子和重金属浓度发生变化. 位于喜马拉雅山中段的冰川 融水补给湖, 由于冰川退缩和融水增加, 导致冰底部环境中的硫化物被氧化释放, 使得湖水中的硫酸根离子 浓度在近 20 年增加了 4 倍, 硫酸根离子浓度与冰川径流量存在显著的正相关关系 ${ }^{[74]}$, 而溶解永由于冰湖颗 粒物的吸附作用致使其浓度降低 ${ }^{[75]}$. 近 20 年来, 在欧洲的阿尔卑斯山脉的两个冰川融水补给湖中, 湖水中 导电率、硫酸盐、钙、镁、二氧化硅等分别增加 $19 、 16 、 8 、 36$ 和 2 倍 ${ }^{[76]}$. 在南美洲的科迪勒拉山系, 热带高山区 冰川退缩引起了水体中 $\mathrm{Pb}$ 和 $\mathrm{Ni}$ 浓度升高, 严重影响了当地的水质状况 ${ }^{[77]}$.

\section{3 冰川融水与冰湖生物}

\section{1 可溶性溶质与冰湖生物}

来源于冰川融水的氮素和 DOM 等可溶性营养物对于山地冰冻圈贫营养冰湖的生态系统来说有着举足 轻重的作用, 其输人量影响浮游植物、细菌的生长率以及群落组成和结构 ${ }^{[45,52]}$ (图 1). 氮素负荷影响着冰湖 生态系统的藻类群落结构, 降低 $\mathrm{pH}$ 值, 改变营养盐的利用率 ${ }^{[78]}$. 冰川融水的氮素影响冰湖中浮游植物的分 布, 冰川补给湖由于融水对湖表层氮素的补给, 浮游植物在表层聚集, 不会在深层出现叶绿素浓度最大 值 ${ }^{[79]}$, 而非冰川融水补给湖则可能在深层出现叶绿素浓度最大值 ${ }^{[6,80]} .20$ 世纪以来冰川融水增加, 导致冰 川补给湖泊氮素的增加, 藻类聚合体发生显著变化 ${ }^{[59]}$, 叶绿素浓度 (如东非的鲁文佐里山脉 ${ }^{[81]}$ 和青藏高 原 ${ }^{[82]}$ 的冰湖) 升高. 一些对氮素富集具有良好指示作用的物种, 如星杆藻属和脆杆藻属, 近 10 年来在冰湖 中生物量呈增加趋势 ${ }^{[79]}$.

目前对于冰川融水补给冰湖中的生物对重金属、POPs 等污染物富集过程及其响应机理的认识相对薄 弱. POPs 能在活的生物体脂肪组织中进行生物积累, 这些有机污染物质还具有生物放大效应, 并通过生物 链逐渐积聚成高浓度, 在冰湖的水生生物中富集. 冰川融水补给的冰湖生物体内有机氯污染物浓度可高达 其他非冰川融水补给湖泊的近千倍 ${ }^{[6]]}$. 此外, 气候变暖将加速生态系统中有机碳的分解, 使得更多与有机 碳结合的 POPs 污染物被释放, 增加了冰湖中可以被生物有效利用的 POPs, 即增大了 POPs 生物可给性 ${ }^{[69]}$. 可见, 持续涌人的高浓度的有机污染物等可溶性性物质会对冰湖物种多样性和稳定性产生显著影响, 影响 强度及其时空尺度与冰湖生态系统的结构和功能、对可溶性物质的敏感性以及与冰川的距离有关.

\section{2 悬移质与冰湖生物}

冰川融水输人的悬移质主要通过改变湖泊的透明度/浊度和温度, 使得冰湖生态系统中浮游生物的生 境条件在时间和空间上产生异质性, 从而对冰湖生物产生影响 (图 1), 冰川融水导致的浊度增加对生物的影 响甚至超过来源于冰川融水氮素和 DOM 等可溶性营养物的影响 ${ }^{[6]}$. 一般来说, PRA 和 UVR 穿透的深度与 湖水浊度呈负相关 ${ }^{[55]}$, 而 PRA 是植物生命活动、有机物质合成和产量形成的能量来源, UVR 则影响固氮过 
程和浮游生物的生产率, 两者的变化均会对水生生物产生显著影响. 冰湖的微生物群落分布特征与光辐射 能密切相关, 因湖水浊度不同而使得 PRA 和 UVR 产生了水平梯度上的变化, 进而影响着微生物分布、生物 量和种类, 甚至出现冰湖的生物群落最终可能被异养的和混合营养的微生物类所占领的现象 ${ }^{[54,83]}$. 另一方 面, 在冰川补给的浑浊的冰湖中, 由于湖水高浓度悬浮颗粒对病毒的潜在消除作用, 湖中病毒性颗粒也会随 着与冰川融水人口距离变小而减少 ${ }^{[84]}$.

冰湖悬移质对湖中不同种类生物多样性的影响存在差异. 冰川融水携带的悬移质可改变冰湖上层和深 层的生态过程、重建湖水能量路径. 一般湖水浊度越高, 浮游生物的多样性就越低 ${ }^{[85-86]}$. 很多浊度较高的冰 湖中, 不仅滤食型枝角类水蚤缺少, 而且其他食性方式蚤类、异养性鞭毛虫的生长也受到限制 ${ }^{[53,83]}$. 冰川融 水注人能导致冰湖细菌群落的多样性增加, 很多细菌类群能够在浑浊冰湖中生存. 在奥地利中部阿尔卑斯 山冰湖细菌种类多样性和发育多样性是随浊度梯度下降而下降的, 但代谢功能多样性却与浊度大小呈负相 关 ${ }^{[87]}$. 新生的浑浊冰湖中生物以原核生物 (细菌) 和后生动物 (轮虫类、线虫等) 为主, 之后浮游生物增加, 如 桡足类和枝角类 (水蚤类) 等, 生物多样性变得丰富 ${ }^{[83]}$. 此外, 冰湖浊度还能改变食物网中生物体的习性. Jönsson 等 ${ }^{[88}$ 发现, 巴塔哥尼亚高原冰湖中本地鱼类在高浊度水平下滤食率减少, 而且高浊度水平还可影响 很多鱼类定位和捕捉猎物的视觉能力,并改变被食者一捕食者的相互作用.

\section{4 问题与不足}

冰川退缩导致湖水量增加已是不争的事实, 并深刻影响山地冰冻圈冰湖的水量变化、理化性质和生态 系统, 成为冰冻圈水文学研究的热点科学问题. 遥感技术是冰川融水与冰湖水资源及灾害效应监测的最有 效手段之一, 但是由于航空/航天测量特定年份、特定季节的瞬时观测值, 高空间分辨率遥感影像的时间分 辨率难以反映冰湖快速变化状态, 往往使得冰湖的季节变化掩盖于年际变化中或者年际变化掩盖于年代际 变化中; 另一方面, 由于冰湖一般规模较小, 中低空间分辨率遥感数据已很难满足冰湖监测的现实需求. 合 成孔径雷达 (SAR) 技术因其能全天候、全天时地提供高分辨率的雷达图像, 有望成为冰湖遥感监测的新手 段,尽管当前基于 SAR 技术还主要应用于冰湖边界得提取上. 总之,山区地形复杂、气象气候条件多变、冰湖 理化性质差异很大, 如何发展传感器准确获取复杂的冰湖信息成为冰湖遥感监测的技术瓶颈之一.

对于冰川融水与湖水量的研究已由本世纪初的关注冰川融水对冰湖面积的变化定性分析, 发展到关注 冰川融水对冰湖水量贡献的定量估算. 当前技术条件很难对冰湖水量进行大范围直接测量, 一般是通过观 测样本冰湖的水深 (水下地形)、面积、体积间回归方程推广计算湖水量,但误差较大,甚至超过 $100 \%{ }^{[89]}$, 而 定量评估冰川融水在湖泊补给作用或补给比重更不多见. 在测量上, 从 $1980 \mathrm{~s}$ 用测量绳进行冰湖水下地形测 量, 发展到基于声呐现场测量冰湖水下地形 ${ }^{[90]}$ 和利用激光测高技术等遥感技术观测湖水位和面积计算湖水 量 $^{[31]}$, 但不太适合对大量、小规模冰湖的监测. 未来将继续发展遥感观测技术和 GIS 空间分析技术, 以更精 准地进行湖水量计算.

由于冰湖多位于不易到达的高寒山区, 当前湖水采样点分布不均, 长时间尺度 (年际年代际) 的冰湖理 化性质定位半定位观测、冰川融水对冰湖生物区 (系) 的直接影响观测等缺乏. 由于环境特殊, 湖水样品采集 及采集后的样品保存技术 (如减缓采集湖水样发生生物作用、化合物水解及氧化还原作用的化学保存技术 等)有更严格的要求. 而且, 对于冰湖中化学成分的来源 (如氮素) 及其与湖水中生物相互作用过程与机理的 认识不足或存在争议 ${ }^{[6]}$, 很多结论都带有地域性特征, 大区域尺度的普适性的研究成果缺乏, 少有冰川融水 及其变化对冰湖水文效应的系统理论、数值模拟与预测预报研究.

冰川被视为大气沉降化学成分储存库和微生物聚集港, 这些成分通过冰川融水中汇集到冰湖中, 从物 理、化学、生物等多方面综合影响冰湖理化性质和生态系统, 影响程度一般与流域冰川的类型、规模和作用 时间有关. 但是融水对冰湖的物理、化学、生物影响程度并非与融水汇人量呈正比,有时甚至很小比例的融 水汇人也能对湖泊生态产生显著影响 ${ }^{[6,77,79]}$. 冰川融水量的变化与冰湖水文效应关系复杂, 冰湖系统对冰川 融水变化响应过程与机理研究有待深人, 湖水面温度等物理属性的变化对水面蒸发、湖水环流、局地环流、 甚至区域气候等观测缺乏, 冰川消失对冰湖水文与生态系统的影响仍知之不多, 冰川融水对冰湖的综合影 响也多停留在定性认识, 定量的耦合观测与模拟研究少见. 此外, 氮素、DOM、POPs、各类离子浓度和重金属 
含量等易在冰湖中聚集, 其理化环境长期深受融水影响,但当前缺乏长时间、大区域尺度的冰川融水对冰湖 理化性质及生态系统的综合影响研究.

\section{5 小结与展望}

山地冰冻圈冰湖是冰川、积雪与冻土三者耦合的连接点, 通过热量、水、物质循环对生态环境与水资源 产生重要影响, 是山地冰冻圈环境变化的敏感指标和前哨, 其中冰川融水及其变化是山地冰冻圈冰湖系统 时空异质性及其变化的驱动器. 当前在喜马拉雅山、天山、阿尔卑斯山、安第斯山、阿拉斯加、东非鲁文佐里 山及青藏高原和巴塔哥尼亚高原等山区典型冰湖开展湖水量、理化性质、生态系统的观测与采样分析, 主要 在以下 3 方面取得进展: (1) 由于冰湖不断接纳冰川融水, 这在一定程度上调整了山地冰冻圈的水循环过 程, 影响冰湖的蓄水量和蓄水周期; 而且, 冰湖潜能因冰川融水不断集聚而增加, 冰湖溃决风险的频次和强 度都将增大. (2) 冰川融水对冰湖物理性质的影响主要表现在降低湖水温度、影响透明度/浊度、改变湖水密 度、造成湖水热力分层现象等方面, 对湖泊化学性质的影响主要表现在增加湖水中的氮素、POPs、各类离子 浓度等. (3) 冰川融水通过改变冰湖物理化学性质来影响湖泊生物. 融水导致湖水温度降低和带来的可溶性 溶质影响浮游生物的分布、组成和结构; 冰川融水输人的悬移质使得冰湖生态系统中浮游生物的生境产生 时空异质性, 湖水中悬移质浓度越高, 浮游生物的多样性越低. 从更长时间尺度来看, 冰川退缩和冰川融水 携带物质汇聚湖中, 使得陆生环境和水生环境信息紧密耦合, 是生物地球化学信息分析载体和理论基础 ${ }^{[61]}$.

当前围绕冰川融水对冰湖水文效应的影响研究仍存在诸多需要探究的科学问题: 冰湖在冰川水资源的 动态变化中在多大程度上扮演什么角色? 冰川融水对冰湖的补给作用和补给比例如何? 多大的冰川融水 汇人量或补给比例阈值能对冰湖水文与生态产生影响? 冰川融水如何在时空与数量 (包括化学和生物地球 化学)上影响冰湖水量 (化学、生物地球化学)平衡? 被滞留的冰川融水能在多大程度上对局地甚至区域的 气象气候、水文过程、生态环境等产生什么样的影响? 现代冰川一冰湖耦合关系 (通过冰川融水汇人) 对现 代冰湖系统的演化有哪些影响? 对古湖泊学研究有哪些启示? 未来冰川退缩甚至消失 (即冰川融水变化) 将对山地冰冻圈湖泊产生什么样的资源、灾害及生态环境效应? 为此, 当前需要深人、系统地开展山地冰冻 圈冰湖的调查、监测、分析等工作, 发展冰湖水文对冰川融水及其变化响应模型, 诊断、评估和预测冰川融水 及变化对冰湖水文与生态环境的效应, 丰富冰川水文与水资源、山地冰冻圈生态环境等领域的科学研究, 促 进冰冻圈科学的发展.

致谢: 感谢本团队研究人员 (张世强、陈仁升、车涛、韩添丁、谢昌卫、上官冬辉、韩海东、何晓波、杨国靖、赵求 东、王生霞、李向应、赵传成、刘俊峰、张伟、陈浩、阳勇、秦甲、许民、王雁) 在本文撰写过程中提出宝贵的修改 意见.

\section{6 参考文献}

[ 1 ] Qin DH, Yao TD, Ding YJ et al eds. Glossary of gryospheric science. Beijing: China Meteorological Press, 2016: 12. [秦 大河, 姚檀栋, 丁永建等. 冰冻圈科学辞典. 北京: 气象出版社, 2016: 12.]

[ 2 ] Yang CD, Wang X, Wei JF et al. Chinese glacial lake inventory based on 3S technology method. Acta Geographica Sinica, 2019, 74(3) : 544-556. [杨成德, 王欣, 魏俊峰等. 基于 3S 技术方法的中国冰湖编目. 地理学报, 2019, 74(3): 544-556.]

[ 3 ] Zhang GQ, Yao TD, Xie HJ et al. An inventory of glacial lakes in the Third Pole region and their changes in response to global warming. Global \& Planetary Change, 2015, 131: 148-157. DOI: 10.1016/j.gloplacha.2015.05.013.

[ 4 ] Wang X, Ding YJ, Liu SY et al. Changes of glacial lakes and implications in Tian Shan, central Asia, based on remote sensing data from 1990 to 2010. Environmental Research Letters, 2013, 8(4) : 575-591. DOI: 10.1088/1748-9326/8/ 4/044052.

[ 5 ] Zhang GQ. Changes in lakes on the Tibetan Plateau observed from satellite data and their responses to climate variations. Progress in Geography, 2018, 37(2) : 214- 223. DOI: 10.18306/dlkxjz.2018.02.004. [张国庆. 青藏高原湖泊变化遥 感监测及其对气候变化的响应研究进展. 地理科学进展, 2018, 37(2) : 214-223.]

[ 6 ] Slemmons KE, Saros JE, Simon K. The influence of glacial meltwater on alpine aquatic ecosystems: a review. Environ Sci 
Process Impacts, 2013, 15(10) : 1794-1806. DOI: 10.1039/c3em00243h.

[ 7 ] Wang X, Liu QH, Liu SY et al. Heterogeneity of glacial lake expansion and its contrasting signals with climate change in Tarim Basin, Central Asia. Environmental Earth Sciences, 2016, 75(8) : 696. DOI: 10.1007/s12665-016-5498-4.

[ 8 ] Wang X, Liu SY, Mo HW et al. Expansion of glacial lakes and its implication for climate changes in the Chinese Himalaya. Acta Geographica Sinica, 2011, 66(7): 895-904. DOI: 10.11821/xb201107003. [王欣, 刘时银, 莫宏伟等. 我国 喜马拉雅山区冰湖扩张特征及其气候意义.地理学报, 2011, 66(7): 895-904.]

[ 9 ] Sorg A, Bolch T, Stoffel M et al. Climate change impacts on glaciers and runoff in Tien Shan (Central Asia). Nature Climate Change, 2012, 2(10): 725-731. DOI: 10.1038/nclimate1592.

[10] Yao TD, Qin DH, Sheng YP et al. Cryospheric changes and their impacts on regional water cycle and ecological conditions in the Qinghai-Tibetan Plateau. Chinese Journal of Nature, 2013, 35(3): 179-186. [姚檀栋, 秦大河, 沈永平等. 青藏 高原冰冻圈变化及其对区域水循环和生态条件的影响.自然杂志, 2013, 35(3) : 179-186.]

[11] Qin DH, Zhou BT, Xiao CD. Progress in studies of cryospheric changes and their impacts on climate of China. Acta Meteorologica Sinica, 2014, (5): 869-879. DOI: 10.11676/qxxb2014.080. [秦大河, 周波涛, 效存德. 冰冻圈变化及其对 中国气候的影响. 气象学报, 2014, (5):869-879.]

[12] Wang NL, Liu SY, Wu QB et al. Recent progress in the study of the change of cryosphere in the northern hemisphere and its impacts on climate and environment. China Basic Science, 2015, (2) : 9-14. [王宁练, 刘时银, 吴青柏等. 北半球 冰冻圈变化及其对气候环境的影响. 中国基础科学, 2015,(2):9-14.]

[13] Duan KQ, Yao TD, Shi PH et al. Simulation and prediction of equilibrium line altitude of glaciers in the eastern Tibetan Plateau. Scientia Sinica Terrae, 2017, 47(1) : 104-113. DOI: 10.1360/N072016-00062. [段克勤, 姚檀栋, 石培宏等. 青藏高原东部冰川平衡线高度的模拟及预测. 中国科学: 地球科学, 2017, 47(1): 104-113.]

[14] Zhang Y, Hirabayashi Y, Fujita K et al. Heterogeneity in supraglacial debris thickness and its role in glacier mass changes of the Mount Gongga. Science China Earth Sciences, 2016, 59(1): 170-184.

[15] Bolch T, Kulkarni A, Kaab A et al. The state and fate of Himalayan glaciers. Science, 2012, 336 (6079) : 310-314. DOI: 10.1126/science.1215828.

[16] Yao TD, Li ZG, Yang W et al. Glacial distribution and mass balance in the Yarlung Zangbo River and its influence on lakes. Chinese Science Bulletin, 2010, 55(18) : 1750-1756. DOI: 10.1007/s11434-010-3213-5. [姚檀栋, 李治国, 杨 威等. 雅鲁藏布江流域冰川分布和物质平衡特征及其对湖泊的影响. 科学通报, 2010, 55(18): 1750-1756.]

[17] Kang SC, Xu YW, You QL et al. Review of climate and cryospheric change in the Tibetan Plateau. Environmental Research Letters, 2010, 5(1) : 015101. DOI: 10.1088/1748-9326/5/1/015101.

[18] Haeberli W, Linsbauer A. Brief communication: Global glacier volumes and sea level - small but systematic effects of ice below the surface of the ocean and of new local lakes on land. The Cryosphere, 2013, 7(3) : 817-821. DOI: 10.5194/tc-7817-2013.

[19] Gardner AS, Moholdt G, Cogley JG et al. A reconciled estimate of glacier contributions to sea level rise: 2003 to 2009. Science, 2013, 340(6134) : 852-857. DOI: 10.1126/science.1234532.

[20] Neckel N, Kropáček J, Bolch T et al. Glacier mass changes on the Tibetan Plateau 2003-2009 derived from ICESat laser altimetry measurements. Environmental Research Letters, 2014, 9(9) : 468-475. DOI: 10.1088/1748-9326/9/1/014009.

[21] Kääb A, Treichler D, Nuth C et al. Brief communication: Contending estimates of 2003-2008 glacier mass balance over the Pamir-Karakoram-Himalaya. The Cryosphere, 2015, 9(2) : 557-564. DOI: 10.5194/tc-9-557-2015.

[22] Brun F, Berthier E, Wagnon P et al. A spatially resolved estimate of High Mountain Asia glacier mass balances, 20002016. Nat Geosci, 2017, 10(9) : 668-673. DOI: 10.1038/NGEO2999.

[23] Wang X, Siegert F, Zhou AG et al. Glacier and glacial lake changes and their relationship in the context of climate change, Central Tibetan Plateau 1972-2010. Global \& Planetary Change, 2013, 111(12) : 246-257. DOI: 10.1016/j. gloplacha.2013.09.011.

[24] Gardelle J, Arnaud Y, Berthier E. Contrasted evolution of glacial lakes along the Hindu Kush Himalaya mountain range between 1990 and 2009. Global \& Planetary Change, 2011, 75(1) : 47-55. DOI: 10.1016/j.gloplacha.2010.10.003.

[25] Nie Y, Sheng YW, Liu Q et al. A regional-scale assessment of Himalayan glacial lake changes using satellite observations from 1990 to 2015. Remote Sensing of Environment, 2017, 189: 1-13. DOI: 10.1016/j.rse.2016.11.008.

[26] Wang WC, Xiang Y, Gao Y et al. Rapid expansion of glacial lakes caused by climate and glacier retreat in the Central Hi- 
malayas. Hydrological Processes, 2014, 29(6) : 859-874.

[27] Wang X, Chai KG, Liu SY et al. Changes of glaciers and glacial lakes implying corridor-barrier effects and climate change in the Hengduan Shan, southeastern Tibetan Plateau. Journal of Glaciology, 2017, 63(239) : 1-8. DOI: 10.1017/jog. 2017.14.

[28] Wang X, Liu SY, Guo WQ et al. Using remote sensing data to quantify changes in Glacial Lakes in the Chinese Himalaya. Mountain Research \& Development, 2012, 32(2) : 203-212. DOI: 10.1659\%2Fmrd-journal-d-11-00044.1.

[29] Wang X, Han HD, Wang J et al. Thermal regime of a supraglacial lake on the debris-covered Koxkar Glacier, southwest Tianshan, China. Environmental Earth Sciences, 2012, 67(1) : 175-183. DOI: 10.1007/s12665-011-1490-1.

[30] Song CQ, Huang B, Richards K et al. Accelerated lake expansion on the Tibetan Plateau in the 2000s: Induced by glacial melting or other processes? Water Resources Research, 2014, 50(4) : 3170-3186. DOI: 10.1002/2013wr014724.

[31] Zhang GQ, Yao TD, Piao SL et al. Extensive and drastically different alpine lake changes on Asia's high plateaus during the past four decades. Geophysical Research Letters, 2017, 44(1) : 252-260. DOI: 10.1002/2016gl072033.

[32] Song CQ, Sheng YW, Ke LH et al. Glacial lake evolution in the southeastern Tibetan Plateau and the cause of rapid expansion of proglacial lakes linked to glacial-hydrogeomorphic processes. Journal of Hydrology, 2016, 540 : 504-514. DOI: 10.1016/j.jhydrol.2016.06.054.

[33] Ju JT, Zhu LP, Wang JB et al. Estimating the contribution of glacial meltwater to Ranwu Lake, a proglacial lake in SE Tibet, using observation data and stable isotopic analyses. Environmental Earth Sciences, 2017, 76(5) : 229. DOI: 10. 1007/s12665-017-6544-6.

[34] Das Pranab Kr. Global warming, glacial lakes and cloud burst events in Garhwal-Kumaon Himalaya: A hypothetical analysis. International Journal of Environmental Science, 2015, 5(4). DOI: 10.6088/ijes.2014050100065.

[35] Loriaux T, Casassa G. Evolution of glacial lakes from the Northern Patagonia Icefield and terrestrial water storage in a sealevel rise context. Global \& Planetary Change, 2013, 102(8) : 33-40. DOI: 10.1016/j.gloplacha.2012.12.012.

[36] Wang X, Liu SY, Ding YJ et al. An approach for estimating the breach probabilities of moraine-dammed lakes in the Chinese Himalayas using remote-sensing data. Natural Hazards and Earth System Sciences, 2012, 12: 3109-3122. DOI: 10. 5194/nhess-12-3109-2012.

[37] Clague JJ, Evans SG. A review of catastrophic drainage of moraine-dammed lakes in British Columbia. Quaternary Science Reviews, 2000, 19(17) : 1763-1783. DOI: 10.1016/s0277-3791(00)00090-1.

[38 ] Fujita K, Sakai A, Takenaka S et al. Potential flood volume of Himalayan glacial lakes. Natural Hazards \& Earth System Sciences, 2013, 13(7) : 1827-1839. DOI: 10.5194/nhess-13-1827-2013.

[39] Harrison S, Kargel JS, Huggel C et al. Climate change and the global pattern of moraine-dammed glacial lake outburst floods. Cryosphere, 2018, 12(4) : 1-28. DOI: 10.5194/tc-2017-203.

[40] Wang X, Liu SY, Ding YJ eds. Research on evaluation method and application of outburst disaster in Hail Lake in Himalayas, China. Beijing: Science Press, 2016: 324. [王欣, 刘时银, 丁永建. 中国喜马拉雅山冰碛湖溃决灾害评价方法 与应用研究. 北京: 科学出版社, 2016: 324.]

[41] Ng FL, Liu SY. Temporal dynamics of a jökulhlaup system. Journal of Glaciology, 2009, 55(192) : 651-665. DOI: 10. 3189/002214309789470897.

[42] Shangguan DH, Ding YJ, Liu SY et al. Quick release of internal water storage in a glacier leads to underestimation of the hazard potential of glacial lake outburst floods from Lake Merzbacher in central Tian shan Mountains. Geophysical Research Letters, 2017, 44(19) : 9786-9795. DOI: 10.1002/2017gl074443.

[43] Edmundson JA, Mazumder A. Regional and hierarchical perspectives of thermal regimes in subarctic, Alaskan lakes. Freshwater Biology, 2002, 47(1) : 1-17. DOI: 10.1046/j.1365-2427.2002.00775.x.

[44] Peter H, Sommaruga R. Alpine glacier-fed turbid lakes are discontinuous cold polymictic rather than dimictic. Inland Waters , 2017, 7(1) : 45-54. DOI: 10.1080/20442041.2017.1294346.

[45] Liu Y, Yao T, Jiao N et al. Microbial community structure in moraine lakes and glacial meltwaters, Mount Everest. Fems Microbiology Letters, 2006, 265(1) : 98-105. DOI: 10.1111/j.1574-6968.2006.00477.x.

[46] Hood E, Berner L. Effects of changing glacial coverage on the physical and biogeochemical properties of coastal streams in southeastern Alaska. Journal of Geophysical Research Biogeosciences, 2009, 114 ( G3 ): 162-174. DOI: 10. 1029/2009jg000971. 
[47] Nelitz MA, Moore RD, Parkinson E. Developing a framework to designate “Temperature Sensitive Streams” in the BC interior. Final report prepared by ESSA Technologies Ltd., University of British Columbia, and BC Ministry of Environment for BC Forest Science Program, Price water house Coopers, Vancouver, BC, 2008: 3.

[48] Moore RD. Stream temperature patterns in British Columbia, Canada, based on routine spot measurements. Canadian Water Resources Journal, 2006, 31(1) : 41-56. DOI: 10.4296/cwrj3101041.

[49] Sugiyama S, Minowa M, Sakakibara D et al. Thermal structure of proglacial lakes in Patagonia. Journal of Geophysical Research Earth Surface, 2016, 121(12) : 2270-2286. DOI: 10.1002/2016jf004084.

[50] Pizarro J, Vergara PM, Cerda S et al. Cooling and eutrophication of southern Chilean lakes. Sci Total Environ, 2016, 541: 683-691. DOI: 10.1016/j.scitotenv.2015.09.105.

[51] Haeberli W, Kääb A, Mühll DV et al. Prevention of outburst floods from periglacial lakes at Grubengletscher, Valais, Swiss Alps. Journal of Glaciology, 2001, 47(156) : 111-122. DOI: 10.3189/172756501781832575.

[52] Van Colen WR, Mosquera P, Vanderstukken M et al. Limnology and trophic status of glacial lakes in the tropical Andes (Cajas National Park, Ecuador). Freshwater Biology, 2016, 62(3) : 458-473. DOI : 10.1111/fwb.12878.

[53] Sommaruga R, Kandolf G. Negative consequences of glacial turbidity for the survival of freshwater planktonic heterotrophic flagellates. Sci Rep, 2014, 4(7) : 4113. DOI: 10.1038/srep04113.

[54] Hylander S, Jephson T, Lebret K et al. Climate-induced input of turbid glacial meltwater affects vertical distribution and community composition of phyto- and zooplankton. Journal of Plankton Research, 2011, 33( 8) : 1239-1248. DOI: 10. 1093/plankt/fbr025.

[55] Rose KC, Hamilton DP, Williamson CE et al. Light attenuation characteristics of glacially-fed lakes. Journal of Geophysical Research Biogeosciences, 2014, 119(7) : 2169-8953. DOI: 10.1002/2014jg002674.

[56] Gallegos CL, Davies-Colley RJ, Gall M. Optical closure in lakes with contrasting extremes of reflectance. Limnology \& Oceanography, 2008, 53(5) : 2021-2034. DOI: 10.4319/lo.2008.53.5.2021.

[57] Bonalumi M, Anselmetti FS, Kaegi R et al. Particle dynamics in high-Alpine proglacial reservoirs modified by pumpedstorage operation. Water Resources Research, 2011, 47(9) : 178-187. DOI: 10.1029/2010wr010262.

[58] Horodyskyj UN. Thermal and physical investigations into lake deepening processes on Spillway Lake, Ngozumpa Glacier, Nepal. Water, 2017, 9: 362. DOI: 10.3390\%2Fw9050362.

[59] Saros JE, Rose KC, Clow DW et al. Melting Alpine glaciers enrich high-elevation lakes with reactive nitrogen. Environmental Science \& Technology, 2010, 44(13) : 4891-4896. DOI: 10.1021/es100147j.

[60] Lafrenière MJ, Sharp MJ. A comparison of solute fluxes and sources from glacial and non-glacial catchments over contrasting melt seasons. Hydrological Processes, 2005, 19(15) : 2991-3012.

[61] Hood E, Fellman J, Spencer RG et al. Glaciers as a source of ancient and labile organic matter to the marine environment. Nature, 2009, 462(7276) : 1044-1047. DOI: 10.1038/nature08580.

[62] Singer GA, Fasching C, Wilhelm L et al. Biogeochemically diverse organic matter in Alpine glaciers and its downstream fate. Nature Geoscience, 2012, 5(5) : 710-714. DOI: 10.1038/ngeo1581.

[63] Hood E, Scott D. Riverine organic matter and nutrients in southeast Alaska affected by glacial coverage. Nature Geoscience, 2008, 1(9) : 583-587. DOI: 10.1038/ngeo280.

[64] Sickman JO, Leydecker AL, Melack JM. Nitrogen mass balances and abiotic controls on N retention and yield in high-elevation catchments of the Sierra Nevada, California, United States. Water Resources Research, 2001, 37( 5 ) : 1445-1461. DOI: $10.1029 / 2000 \mathrm{wr} 900371$.

[65] Blais JM, Schindler DW, Muir DC et al. Melting glaciers: a major source of persistent organochlorines to subalpine Bow Lake in Banff National Park, Canada. Ambio, 2001, 30(7) : 410-415. DOI: 10.1579/0044-7447-30.7.410.

[66] Blais JM, Schindler DW, Muir DCG et al. Accumulation of persistent organochlorine compounds in mountains of western Canada. Nature, 1998, 395(6702) : 585-588. DOI: 10.1038/26944.

[67] Bettinetti R, Quadroni S, Galassi S et al. Is meltwater from Alpine glaciers a secondary DDT source for lakes? Chemosphere, 2008, 73(7) : 1027-1031. DOI: 10.1016/j.chemosphere.2008.08.017.

[68] Cheng H, Lin T, Zhang G et al. DDTs and HCHs in sediment cores from the Tibetan Plateau. Chemosphere, 2014,94 (1) : 183-189. DOI: 10.1016/j.chemosphere.2013.10.012.

[69] Wang XP, Sun DC, Yao TD. Climate change and global cycling of persistent organic pollutants: A critical review. Scientia Sinica Terrae, 2016, (10) : 1301-1316. DOI: 10.1007/s11430-016-5073-0. [王小萍, 孙殿超, 姚檀栋. 气候变化与 
持久性有机污染物全球循环. 中国科学: 地球科学, 2016, (10): 1301-1316.]

[70] Schmid P, Bogdal C, Blüthgen N et al. The missing piece: sediment records in remote Mountain lakes confirm glaciers being secondary sources of persistent organic pollutants. Environmental Science \& Technology, 2011, 45(1) : 203-208. DOI: 10.1021/es1028052.

[71] Pavlova PA, Zennegg M, Anselmetti FS et al. Release of PCBs from Silvretta glacier (Switzerland) investigated in lake sediments and meltwater. Environ Sci Pollut Res Int, 2016, 23(11) : 10308-10316. DOI: 10.1007/s11356-015-5854-z.

[72] Bettinetti R, Galassi S, Guilizzoni P et al. Sediment analysis to support the recent glacial origin of DDT pollution in Lake Iseo ( Northern Italy). Chemosphere, 2011, 85(2) : 163-169. DOI: 10.1016/j.chemosphere.2011.06.037.

[73] Bogdal C, Schmid P, Zennegg M et al. Blast from the past: melting glaciers as a relevant source for persistent organic pollutants. Environmental Science \& Technology, 2009, 43(21) : 8173-8177. DOI: 10.1021/es901628x.

[74] Salerno F, Rogora M, Balestrini R et al. Glacier melting increases the solute concentrations of Himalayan Glacial Lakes. Environ Sci Technol, 2016, 50(17) : 9150-9160. DOI: 10.1021/acs.est.6b02735.

[75] Sun S, Kang S, Huang J et al. Distribution and transportation of mercury from glacier to lake in the Qiangyong Glacier Basin, southern Tibetan Plateau, China. J Environ Sci, 2016, 44(6) : 213-223. DOI: 10.1016/j.jes.2015.09.017.

[76] Thies H, Nickus U, Mair V et al. Unexpected response of high Alpine Lake waters to climate warming. Environ Sci Techn$o l, 2007,41(21)$ : 7424-7429. DOI: 10.1021\%2Fes0708060.

[77] Fortner SK, Mark BG, Mckenzie JM et al. Elevated stream trace and minor element concentrations in the foreland of receding tropical glaciers. Applied Geochemistry, 2011, 26(11) : 1792-1801. DOI: 10.1016/j.apgeochem.2011.06.003.

[78] Baron JS, Schmidt TM, Hartman MD et al. Climate-induced changes in high elevation stream nitrate dynamics. Global Change Biology, 2009, 15(7) : 1777-1789. DOI: 10.1111/j.1365-2486.2009.01847.x.

[79] Saros JE, Interlandi SJ, Doyle S et al. Are the deep chlorophyll maxima in alpine lakes primarily induced by nutrient availability, not UV Avoidance? Arctic Antarctic \& Alpine Research, 2005, 37 (4) : 557-563. DOI: 10.1657/1523-0430 (2005) 037[0557: ATDCMI $] 2.0 . \mathrm{CO} ; 2$.

[80] Slemmons KEH, Saros JE. Implications of nitrogen-rich glacial meltwater for phytoplankton diversity and productivity in alpine lakes. Limnology and Oceanography, 2012, 57(6) : 1651-1663. DOI: 10.4319/lo.2012.57.6.1651.

[81] Mcglynn G, Mackay AW, Rose NL et al. Palaeolimnological evidence of environmental change over the last 400 years in the Rwenzori Mountains of Uganda. Hydrobiologia, 2010, 648(1) : 109-122. DOI: 10.1007/s10750-010-0144-8.

[82] Lami A, Turner S, Musazzi S et al. Sedimentary evidence for recent increases in production in Tibetan plateau lakes. Hydrobiologia, 2010, 648(1) : 175-187. DOI: 10.1007/s10750-010-0263-2.

[83] Sommaruga R. When glaciers and ice sheets melt: consequences for planktonic organisms. Journal of Plankton Research, 2015, 37(3) : 509. DOI: 10.1093\%2Fplankt \% 2Ffbv027.

[84] Fabian D, Hannes P, Ruben S. Are viruses important in the plankton of highly turbid glacier-fed lakes? Scientific Reports, 2016, 6: 24608. DOI: 10.1038/srep24608.

[85] Koenings JP, Burkett RD, Edmundson JM. The exclusion of limnetic cladocera from turbid glacier-meltwater lakes. Ecology, 1990, 71(1) : 57-67. DOI: 10.2307/1940247.

[86] Donohue I, Garcia MJ. Impacts of increased sediment loads on the ecology of lakes. Biological Reviews, 2009, 84(4): 517-531. DOI: 10.1111/j.1469-185x.2009.00081.x.

[87] Peter H, Sommaruga R. Shifts in diversity and function of lake bacterial communities upon glacier retreat. The ISME Journal, 2016, 10(7) : 1545-1554. DOI: 10.1038/ismej.2015.245.

[88] Jönsson M, Ranåker L, Nicolle A et al. Glacial clay affects foraging performance in a Patagonian fish and cladoceran. Hydrobiologia, 2011, 663(1) : 101-108. DOI : 10.1007/s10750-010-0557-4.

[89] Cook SJ, Quincey DJ. Estimating the volume of Alpine glacial lakes. Earth Surface Dynamics Discussions, 2015, 3(3): 909-940. DOI: 10.5194/esurfd-3-909-2015.

[90] Yao XJ, Liu SY, Sun MP et al. Volume calculation and analysis of the changes in moraine-dammed lakes in the north Himalaya: a case study of Longbasaba Lake. Journal of Glaciology, 2012, 58(210) : 753-760.

[91] Engstrom DR, Fritz SC, Almendinger JE et al. Chemical and biological trends during lake evolution in recently deglaciated terrain. Nature, 2000, 408(6809) : 161-166. DOI: 10.1038/35041500. 